\title{
The Role of Tofacitinib in the Management of Alopecia Totalis
}

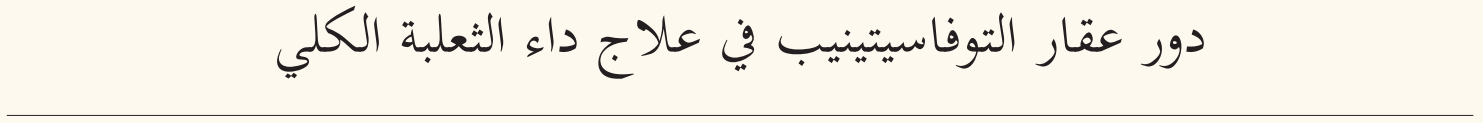

كارلوس هرنانديز مونتويا و ريكاردو رويز-فيلافيردي
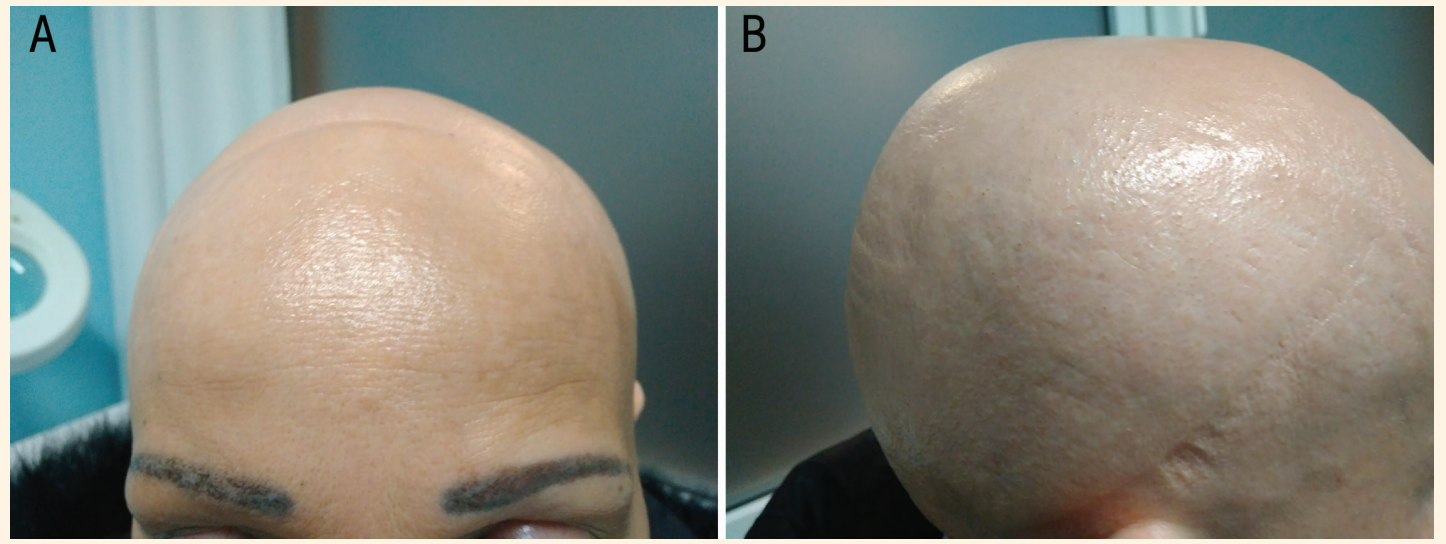

Figure 1: Photographs of the scalp of a 37-year-old female patient showing alopecia totalis, which is an advanced form of alopecia areata.

A 37-YEAR-OLD CAUCASIAN FEMALE PATIENT was referred to the dermatology unit of the Hospital de Poniente, El Ejido, Spain, in 2017 with alopecia totalis (AT). The AT had occurred over the past five months and was classified as grade IV (75-100\% hair loss) severity alopecia tool index (SALT) [Figure 1]. No nail changes were found prior to treatment.

Previous failure of topical treatments (high potency corticosteroids, diphenylcyclopropenone and minoxidil $5 \%$ ), infiltration of intralesional corticosteroids (triamcinolone acetonide/mepivacaine $2 \%$ at $2 \mathrm{~mL} /$ month for six months) and dexamethasone minipulses $(0.1 \mathrm{mg} / \mathrm{kg}$ twice a week for six months) was reported. There was no personal or family history of alopecia areata. Following approval of the hospital's pharmaceutical committee, treatment with tofacitinib $5 \mathrm{mg} / 12$ hours was prescribed five months after the last unsuccessful treatment (dexamethasone minipulses). Complete blood count, liver and kidney function tests, electrolytes, thyroid function, serology tests for hepatitis B and C, HIV and tuberculin skin test were within normal ranges.

A $40 \%$ regrowth of the hair of the scalp was observed at three months [Figure 2]. At six months, the patient's remaining hair loss was equivalent to SALT I (less than $25 \%$ total hair loss) with a substantial improvement of her quality of life as recored on the dermatology quality of life index from 25 to 3 [Figure 3]. Routine biochemical tests, such as hepatic and kidney function tests and lipid profile showed no anomalies during the course of the treatment. The patient is currently continuing treatment with no side effects reported for the previous 10 months.

\section{Comment}

Tofacitinib is an inhibitor of Janus kinase- 3 and is approved by the Food and Drug Administration for the treatment of rheumatoid arthritis (RA). Craiglow and King first observed the effectiveness of tofacitinib in the treatment of alopecia areata. ${ }^{1}$ Subsequently, retrospective studies with more than 90 patients and clinical trials using the dosage approved for RA evaluated its short- and medium-term effectiveness. ${ }^{2}$ Alopecia areata is considered an autoimmune disease with a $1.7 \%$ incidence rate. Scalp biopsy will not help in determining the cause of alopecia in such patients. 


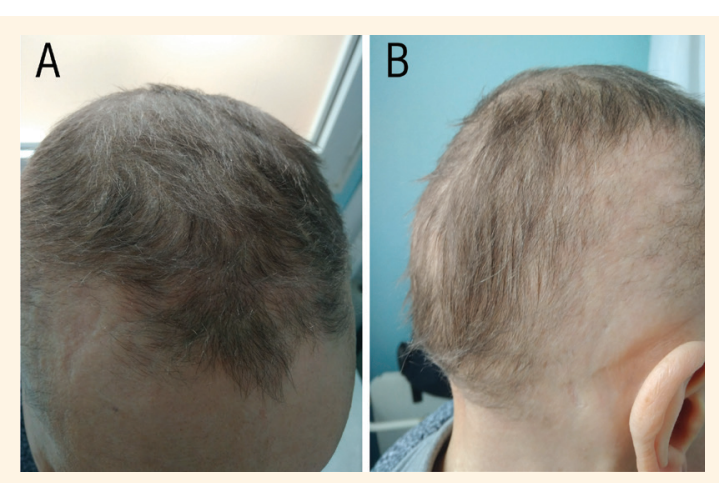

Figure 2: Photographs of the scalp of a 37-year-old female patient after three months of treatment with tofacitinib.

Treatment with tofacitinib may be adapted according to previously reported cases. ${ }^{3}$ Frequent side effects include grade I and II infections, transaminitis and temporary cholesterol elevations. Nevertheless, doses greater than $10 \mathrm{mg}$ twice a day may be associated with an increased risk of serious infections and malignancies. ${ }^{2}$ The SALT score will be imperative to determine the severity and monitor the response to treatments, using either the original SALT I or SALT II (determined by adding the percentage of hair loss in various areas of the scalp).

Analysis of gene expression can be crucial to identify non-responders and slow responders and adjust

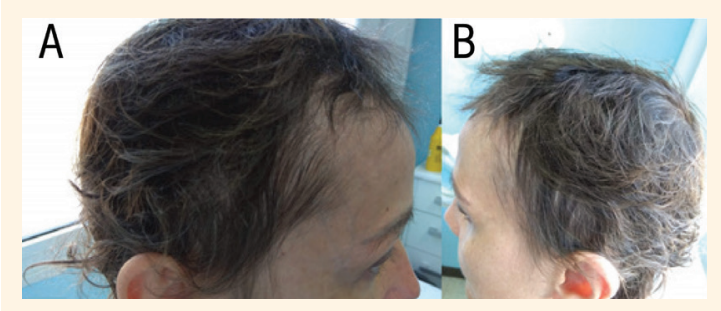

Figure 3: Photographs of the scalp of a 37-year-old female patient after six months of treatment with tofacitinib.

the treatment in a timely manner. Further research to determine protocols of use, monotherapy and combined therapy and monitoring the side effects of alopecia areata are required. Unfortunately, the discontinuation of tofacitinib can cause relapse of the alopecia. ${ }^{3}$

\section{References}

1. Craiglow BG, King BA. Tofacitinib for the treatment of alopecia areata in preadolescent children. J Am Acad Dermatol 2019; 80:568-70. https://doi.org/10.1016/j.jaad.2018.08.041.

2. Iorizzo M, Tosti A. Emerging drugs for alopecia areata: JAK inhibitors. Expert Opin Emerg Drugs. 2018; 23:77-81. https://doi.org/10. 1080/14728214.2018.1444750.

3. Kostovic K, Gulin SJ, Mokos ZB, Ceovic R. Tofacitinib, an oral Janus kinase inhibitor: Perspectives in dermatology. Curr Med Chem 2017; 24:1158-67. https://doi.org/10.2174/18744672106 66170113104503 